Article

\title{
Novel Intact Polar and Core Lipid Compositions in the Pyrococcus Model Species, P. furiosus and $P$. yayanosii, Reveal the Largest Lipid Diversity Amongst Thermococcales
}

\author{
Maxime Tourte ${ }^{1,2}{ }^{\oplus}$, Vanessa Kuentz ${ }^{3,+}$, Philippe Schaeffer ${ }^{3}{ }^{(D}$, Vincent Grossi $\left.{ }^{4}{ }^{(}\right)$, \\ Anais Cario ${ }^{2,4,+}$ and Philippe M. Oger $2, *$ (i) \\ 1 Univ Lyon, Univ Lyon 1, CNRS, UMR 5240, F-69622 Villeurbanne, France; maxime.tourte@univ-lyon1.fr \\ 2 Univ Lyon, INSA Lyon, CNRS, UMR 5240, F-69621 Villeurbanne, France; anais.cario@icmcb.cnrs.fr \\ 3 Univ Strasbourg, CNRS, UMR 7177 Strasbourg, France; v.kuentz@group-irsea.com (V.K.); \\ p.schaef@unistra.fr (P.S.) \\ 4 Univ Lyon, Univ Lyon 1, ENSL, CNRS, LGL-TPE, F-69622 Villeurbanne, France; vincent.grossi@univ-lyon1.fr \\ * Correspondence: philippe.oger@insa-lyon.fr; Tel.: +33-04-72-43-36-01 \\ † Present adresses: Institut de Recherche en Sémiochimie et Ethologie Appliquée F-84400, Apt, France, Univ \\ Bordeaux, CNRS, UMR 5026 F-33600, Pessac, France.
}

Received: 21 April 2020; Accepted: 25 May 2020; Published: 29 May 2020

\begin{abstract}
Elucidating the lipidome of Archaea is essential to understand their tolerance to extreme environmental conditions. Previous characterizations of the lipid composition of Pyrococcus species, a model genus of hyperthermophilic archaea belonging to the Thermococcales order, led to conflicting results, which hindered the comprehension of their membrane structure and the putative adaptive role of their lipids. In an effort to clarify the lipid composition data of the Pyrococcus genus, we thoroughly investigated the distribution of both the core lipids (CL) and intact polar lipids (IPL) of the model Pyrococcus furiosus and, for the first time, of Pyrococcus yayanosii, the sole obligate piezophilic hyperthermophilic archaeon known to date. We showed a low diversity of IPL in the lipid extract of $P$. furiosus, which nonetheless allowed the first report of phosphatidyl inositol-based glycerol mono- and trialkyl glycerol tetraethers. With up to 13 different CL structures identified, the acid methanolysis of Pyrococcus furiosus revealed an unprecedented CL diversity and showed strong discrepancies with the IPL compositions reported here and in previous studies. By contrast, P. yayanosii displayed fewer CL structures but a much wider variety of polar heads. Our results showed severe inconsistencies between IPL and CL relative abundances. Such differences highlight the diversity and complexity of the Pyrococcus plasma membrane composition and demonstrate that a large part of its lipids remains uncharacterized. Reassessing the lipid composition of model archaea should lead to a better understanding of the structural diversity of their lipidome and of their physiological and adaptive functions.
\end{abstract}

Keywords: archaeal membrane lipids; Pyrococcus; P. furiosus; P. yayanosii; phospholipids; glycerophospholipids; core lipids; tetraethers

\section{Introduction}

Archaea are ubiquitous in all Earth ecosystems due to peculiar physiological features that allow them to withstand environmental conditions ranging from the mildest to the harshest. One of these peculiarities lies in the structure of their cell membranes, which are composed of lipids of divergent structures and properties from the typical fatty-acyl phospho- and glyco-lipids found 
in Bacteria and Eukarya. The hydrophobic core of archaeal lipids is made of $\mathrm{C}_{20}, \mathrm{C}_{25}$ and $\mathrm{C}_{40}$ isoprenoid hydrocarbon chains linked to a glycerol backbone in an sn-2,3 configuration by ether bonds, creating cell membranes with enhanced impermeability and stability compared to that of Bacteria and Eukarya [1,2]. Archaeal lipids are classified into two groups: diethers that form membrane bilayers and membrane-spanning tetraethers that form monolayer membranes. The structural core lipid diversity includes Mono- and Dialkyl Glycerol Diethers (MGD and DGD respectively) [3], Glycerol Mono-, Dior Trialkyl Glycerol tetraethers (GMGT, GDGT and GTGT, respectively) [4], di- and tetraethers with hydroxylated or unsaturated isoprenoid chains [5,6] and tetraethers with glycerol, butanol, pentanol and nonitol backbones [7]. The polar head group diversity of archaea mostly resembles that typical for bacterial/eukaryal lipids, with the existence of phospho- and glyco-lipids deriving from sugars [8], aminoacids [9] or combinations of both [9].

The occurrence and distribution of archaeal lipids still remain poorly characterized, partly because the classical extraction procedures may lead to the preferential extraction of some classes of lipids over others. Current data on archaeal lipids might thus not fully represent the real diversity and distribution in the original samples $[10,11]$. Indeed, reinvestigation of the lipid composition of several archaeal isolates using alternative procedures has almost systematically led to the discovery of novel major lipid structures. In this respect, the cases of Methanothermobacter thermautotrophicus $[4,12,13]$ and Thermococcus barophilus [11,14] are noteworthy. Deciphering the precise and complete spectrum of archaeal lipids is now of paramount importance in order to grasp their exact biological relevance, i.e., their physiological and adaptive functions, and to explain the physico-chemical properties of the archaeal membrane under the extreme conditions Archaea most often thrive in.

Pyrococcus furiosus is a piezosensitive ( $<10 \mathrm{MPa})$, hyperthermophilic $\left(70-113^{\circ} \mathrm{C}\right.$, optimal $\left.100{ }^{\circ} \mathrm{C}\right)$, neutrophilic (pH 5.0-9.0, optimal pH 6.8) marine (0.5-5.0\% NaCl, optimal 2.0-3.0\% NaCl) archaeon that was isolated from geothermally heated sediments from Vulcano Island, Italy [15]. Since it is one of the few Archaea for which a genetic system is available [16,17], P. furiosus is now the best-studied species from the Thermococcales order. P. woesei, another Pyrococcus species named after the father of Archaea, Carl Woese, was isolated in the same year from the same location as P. furiosus [18]. From the physiological and genomic points of view, both species are almost identical, as they grow optimally under the exact same conditions of temperature, salinity and $\mathrm{pH}[15,18]$, and exhibit $98 \%$ identity at the genome level and share most of their genomic markers [19]. However, reports of their membrane lipid compositions have shown drastic differences. Indeed, $P$. woesei was shown to produce only DGD with a phosphatidyl inositol head group [20], whereas divergent membrane lipid compositions were reported for P. furiosus [21,22]. Sprott et al. first identified ether lipids with the typical DGD and GDGT hydrophobic cores associated with diverse polar head groups, including both phosphoand glyco-lipids [21]. However, more recently, Lobasso et al. identified novel core lipid structures in P. furiosus, namely GDGT with two cyclopentane rings and an ether lipid cardiolipin [22]. Additionally, the core lipid compositions of P. furiosus and P. woesei differ greatly from that of other Pyrococcus members, such as $P$. abyssi, which synthesizes $15 \%$ of DGD and $85 \%$ of GDGT, including ca. $9 \%$ with two cyclopentane rings [23], P. horikoshii, which mostly produces tetraether lipids ( $>90 \%)$ with more than $15 \%$ in the GMGT monoalkyl form [24-26], and P. kukulkanii, which seemingly produces only diether lipids [27].

In an effort to further explore the membrane lipid composition in the Pyrococcus genus, we here rigorously assayed the intact polar lipid (IPL) and core lipid (CL) compositions of two emblematic species of this genus, P. furiosus, the first isolated and best characterized Pyrococcus species [15], and P. yayanosii, its most piezophilic member, whose lipids are yet unknown [28]. In addition, as it is now well established that archaeal lipid extractions and analyses can be biased [10,11], we used Thermococcus kodakarensis, for which the most complete IPL and CL compositions have been described [29], as a quality control for our lipid extraction procedure and analysis. We show that $P$. yayanosii synthesizes phosphatidylinositol-based IPL with typical DGD and GDGT cores, while several novel IPL and CL could be identified in P. furiosus, notably GMGT and GDGT with up to four cyclopentane rings. 


\section{Materials and Methods}

\subsection{Microorganisms and Growth Conditions}

Pyrococcus furiosus strain DSM3638 was isolated from geothermally heated sediments from the beach of Porto di Levante, Vulcano Island, Italy [15]. Thermococcus kodakarensis strain KOD1 was isolated from a solfatara in a wharf of Kodakara Island, Kagoshima, Japan [30]. Both strains were purchased from the Deutsche Sammlung für Mikroorganismen und Zellkulturen (DSMZ), Braunschweig, Germany. Pyrococcus yayanosii strain CH1 was isolated from the $4100 \mathrm{~m}$ deep Ashadze hydrothermal vent, on the mid-Atlantic ridge [28]. P. yayanosii was obtained from the UBOCC (Université de Bretagne Occidentale-Type Culture Collection, Brest, France). Cultures were grown under strict anaerobiosis in a rich medium established for Thermococcales [28], containing $3 \% \mathrm{NaCl}$ and $10 \mathrm{~g} \cdot \mathrm{L}^{-1}$ elemental sulfur, at $\mathrm{pH}$ 6.8. P. furiosus and T. kodakarensis were cultivated at atmospheric pressure and 98 and $85^{\circ} \mathrm{C}$, respectively, whereas $P$. yayanosii was grown at $52 \mathrm{MPa}$ and $98^{\circ} \mathrm{C}$. The medium was reduced by adding $\mathrm{Na}_{2} \mathrm{~S}(0.1 \%$ w/v final) before inoculation. Growth was monitored by counting with a Thoma cell (depth $0.01 \mathrm{~mm}$ ) using a light microscope (life technologies EVOS ${ }^{\circledR}$ XL Core $400 \times$, Waltham, MA, USA).

Cells of 250-mL cultures in late exponential phase were recovered by centrifugation $(4000 \times g$, $\left.45 \mathrm{~min}, 4^{\circ} \mathrm{C}\right)$ and rinsed twice with an isotonic saline solution $(3 \% \mathrm{w} / \mathrm{v} \mathrm{NaCl})$. Cultivation under high hydrostatic pressure being much more demanding, a significantly lower biomass was recovered for P. yayanosii than for P. furiosus and T. kodakarensis. The cell pellets were lyophilized overnight and kept at $-80^{\circ} \mathrm{C}$ until lipid extraction.

\subsection{IPL Extraction and HPLC-ESI-MS Analysis}

IPL were extracted using a modified Bligh and Dyer (B\&D) method [31]. Briefly, dried cells were extracted with a monophasic mixture of methanol/dichloromethane/purified water $\left(\mathrm{MeOH} / \mathrm{DCM} / \mathrm{H}_{2} \mathrm{O}\right.$; 1:2.6:0.16; $v / v / v)$ using a sonication probe for $15 \mathrm{~min}$. After centrifugation $(2500 \times g, 5 \mathrm{~min})$, the supernatant was collected and the extraction procedure was repeated twice. The supernatants were pooled, dried under reduced pressure and solubilized in Methanol/dichloromethane (DCM) (1:5; $v / v)$. A significant amount of sulfur from the growth medium was extracted alongside archaeal lipids, and the total lipid dry mass of the three Thermococcales species was thus not estimated. IPL were analyzed using high-performance liquid chromatography coupled with electrospray ionization mass spectrometry (HPLC-ESI-MS) using an HP 1100 series HPLC instrument equipped with an auto-injector and a Chemstation chromatography manager software. Separation was achieved on a Diol $5 \mu \mathrm{m}$ column $\left(250 \mathrm{~mm} \times 2.1 \mathrm{~mm}\right.$, Inertsil ${ }^{\circledR}$, GL Science, Torrance, CA, USA) maintained at $30^{\circ} \mathrm{C}$. The injection volume was set to $5 \mu \mathrm{L}$. Di- and tetraether IPL were eluted in the same run with a flow rate of $0.2 \mathrm{~mL} \cdot \mathrm{min}^{-1}$, using the nonlinear gradient described by Sinninghe Damsté et al. [32]. Detection was achieved using an Esquire 3000+ ion trap mass spectrometer with an electrospray ionization source in positive and negative modes. The conditions for the MS analyses were as follows: nebulizer pressure 30 psi, cone tension $40 \mathrm{~V}$, drying gas $\left(\mathrm{N}_{2}\right)$ flow $8 \mathrm{~L} \cdot \mathrm{min}^{-1}$ and temperature $340{ }^{\circ} \mathrm{C}$, capillary voltage $5 \mathrm{kV}$ (negative mode) and $-4 \mathrm{kV}$ (positive mode), mass range $m / z$ 650-2000. IPL structures were determined using Bruker Data Analysis software by comparing the obtained protonated molecular ion masses (and their $\mathrm{NH}_{4}{ }^{+}$and $\mathrm{Na}^{+}$adducts) and specific fragmentation patterns with previously described ones [22,29]. Identified structures and their respective protonated (positive mode) and deprotonated (negative mode) molecular ion masses are displayed in Figure 1. For each archaeal species, IPL relative abundances were determined by integration of the peak area on the mass chromatograms corresponding to the protonated, ammoniated and sodiated adducts in positive mode and to the deprotonated adduct in negative mode. As no archaeal GDGT-based phosphoglycolipid was commercially available, no relevant standard could be used to assay the response factors of the detected IPL. Although different response factors are to be expected in ESI-MS (ElectroSpray Ionizatio-Mass Spectroscopy), notably for lipids bearing distinct classes of polar head groups, a response factor of 1 was arbitrarily chosen for comparison of the relative IPL abundances between the three species investigated. 


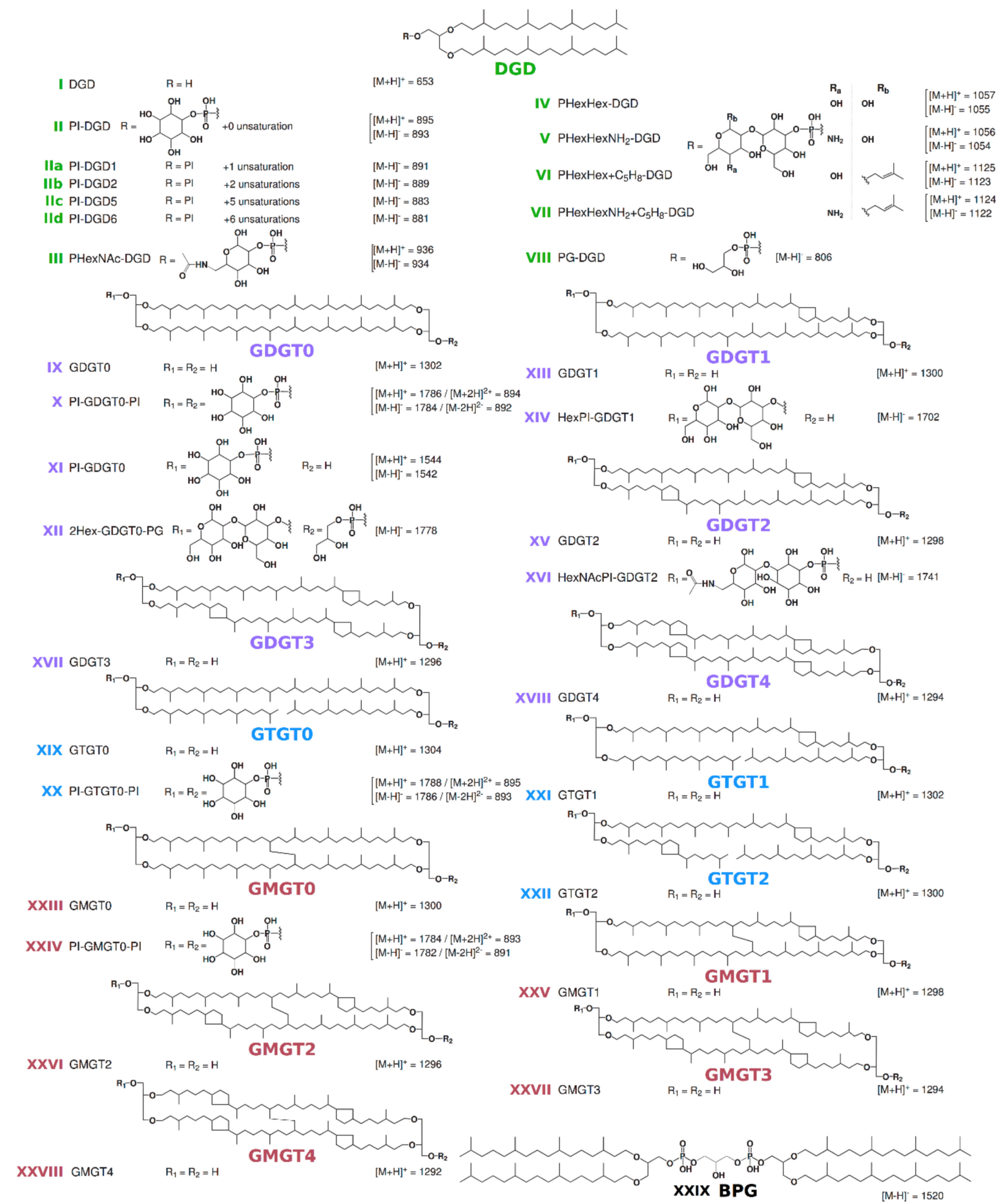

Figure 1. Core and intact polar lipids identified in Pyrococcus furiosus, Pyrococcus yayanosii and Thermococcus kodakarensis and other lipids mentioned in the text. Shorthand nomenclature is indicated. The protonated and/or deprotonated mass-charge ratios are indicated for each structure when detected. Ammoniated and sodiated adducts were also detected and used for integration, but are not represented here. Core structures: dialkyl glycerol diethers (DGD; green) with 0 (I, II, III, IV, V, VI, VII and VIII) and with 1 (IIa), 2 (IIb), 5 (IIc) and 6 (IId) unsaturations; glycerol dialkyl glycerol tetraethers (GDGT; purple) with 0 (IX, X, XI and XII) and with 1 (XIII and XIV), 2 (XV and XVI), 3 (XVII) and 4 cyclopentane rings (XVIII); glycerol trialkyl glycerol tetraethers (GTGT; blue) with 0 (XIX and XX) and with 1 and 2 cyclopentane rings (XXI and XXII); glycerol monoalkyl glycerol tetraethers (GMGT, red) with 0 (XXIII and XXIV) and with 1 to 4 cyclopentane rings (XXV to XXVIII). Polar head groups: 
phosphatidyl inositol (PI, II, IIa to IId, X, XI, XIV, XVI, XX and XXIV), (phosphatidyl) $\mathrm{N}$-acetylhexoseamine ((P)HexNAc, III and XVI), glycosylated phosphatidyl hexose (PHexHex, IV and XIV), PHexHex bearing an additional mass of $68\left(\mathrm{PHexHex}+\mathrm{C}_{5} \mathrm{H}_{8}, \mathbf{V I}\right)$, ammoniated PHexHex $\left(\mathrm{PHexHexNH}_{2}, \mathbf{V}\right)$, PHexHexNH $\mathrm{N}_{2}$ bearing an additional mass of $68\left(\mathrm{PHexHexNH}_{2}+\mathrm{C}_{5} \mathrm{H}_{8}, \mathbf{X}\right)$, phosphatidyl glycerol (PG, VIII and XII), dihexose (2Hex, XII), PI bearing an additional HexNAc head group (HexNAcPI, XIV). In addition, the peculiar structure of an ether cardiolipin gathering two DGD linked by a bisphosphatidyl glycerol (BPG, XXIX) is represented. Positions of cyclopentane rings, covalent bound between the two alkyl chains and additional groups on the polar heads are drawn arbitrarily in this Figure.

\subsection{Preparation and HPLC-APCI-MS Analysis}

In order to exhaustively analyze the CL composition of the strains, polar head groups were removed using acid methanolysis $\left(1.2 \mathrm{~N} \mathrm{HCl}\right.$ in $\mathrm{MeOH}$ at $110{ }^{\circ} \mathrm{C}$ for $3 \mathrm{~h}$ ) of (1) the total lipid extracts, (2) the cell residue obtained after lipid extraction and (3) the intact cell pellets (26). Because of the low biomass of $P$. yayanosii, the CL preparation was conducted only on the total lipid extract and the cell residue for this species. The hydrolyzed lipids were extracted using $\mathrm{MeOH} / \mathrm{DCM}(1: 1, v / v ; \times 3)$ and filtrated over celite. The solvent of the resulting CL extracts was removed under reduced pressure, and the extracts were resolubilized in $n$-hexane/isopropanol $(99: 1, v / v)$. CL analysis was conducted using HPLC coupled with atmospheric-pressure chemical ionization MS (HPLC-APCI-MS) as described in Tourte et al. [26]. Under our analytical conditions, DGD was the only form of diether CL detected, whereas numerous tetraether structures were identified, namely GDGT, GTGT and GMGT and their cyclopentane ring-containing derivatives. A chemically synthesized core DGD and a core GDGT with no cyclopentane ring (GDGT0) isolated from a culture of $M$. thermautotrophicus were used to determine the response factors of the different CL under our analytical conditions. The analysis of a $2 / 1$ standard solution (DGD/GDGT0, \% mol) led to a molar response factor of DGD that was ca. 10 times lower than that of GDGT0. Similarly, to what is frequently found in the literature, a response factor of 1 was assumed between GDGT0 and the other tetraethers. For each archaeal species, CL relative abundances were determined by integration of the peak area on the mass chromatograms corresponding to the protonated adduct only, and the relative abundance of DGD was corrected by a factor of 10 relative to that of tetraethers.

\section{Results}

The analysis of the total lipid extracts from Pyrococcus furiosus, P. yayanosii and Thermococcus kodakarensis obtained with our modified B\&D extraction procedure showed various IPL structures (roman numbers hereafter refer to the structures presented in Figure 1) and distinct lipid compositions in these three closely related hyperthermophilic archaea (Figure 2). P. furiosus IPL were strongly dominated by a DGD with a phosphatidyl inositol polar head group (PI-DGD, compound II, [M-H]- 893; ca. 59\% and $84 \%$ in positive and negative ion modes, respectively; Figure $2 \mathrm{~A}, \mathrm{~B}$ and Table 1 ). Minute amounts of putative derivatives of PI-DGD II were further observed in negative mode. The deprotonated molecular ions detected ([M-H] $]^{-}$891, 889, 883 and 881) could correspond to homologues of PI-DGD II with 1, 2, 5 and 6 unsaturations (compounds IIa to IId), respectively, but the absence of clear fragmentation patterns however impeded their unambiguous identification. Three additional compounds (compounds $X$, XX and XXIV) with different retention times and masses (1788, 1786 and 1784, respectively) likely corresponding to tetraethers, were also detected on the HPLC-ESI-MS chromatogram of P. furiosus. With a predominant $[\mathrm{M}+\mathrm{H}]^{+}$ion at $m / z$ 1786, compound $\mathrm{X}$ was identified as PI-GDGT0-PI. The two other compounds (XX and XXIV) exhibited $[\mathrm{M}+\mathrm{H}]^{+}$ions shifted respectively upwards and downwards by two mass units. The hydrolysis experiments carried out on the solvent extract, the pre-extracted cells and the whole biomass of $P$. furiosus systematically yielded CL dominated by GTGT0 XIX, GDGT0 IX and GMGT0 XXIII (Figure 3A-C). Compounds XX and XXIV were thus tentatively identified as PI-GTGT0-PI and PI-GMGT0-PI, respectively. Confusion with ring-containing homologues was not 
possible due to the well-differentiated retention times observed between acyclic and cyclic tetraether CL (Figure 3A-C). Interestingly, compound XXIV appeared as a doublet of peaks likely corresponding to isomers (Figure 2), as did GMGT0 XXIII released by methanolysis (Figure 3A-C), which further supported the PI-GMGT0-PI structure proposed for compound XXIV. Assuming a response factor of 1 in HPLC-ESI-MS between the different tetraether IPL, PI-GMGT0-PI XXIV was the most abundant tetraether lipid in our modified B\&D extract from P. furiosus, representing ca. $28 \%$ and $11 \%$ of the total IPL in positive and negative ion modes, respectively, whereas PI-GDGT0-PI X and PI-GTGT-PI XX only reached ca. $8 \%$ and $2 \%$ and ca. $5 \%$ and $1 \%$ of the total IPL, respectively (Figure $2 \mathrm{~A}, \mathrm{~B}$ and Table 1). In addition, small amounts of DGD with a phosphatidyl N-acetylhexosamine polar head group (PHexNAc-DGD; compound III, ca. 2\% in negative mode) were detected in P. furiosus (Figure 2A,B and Table 1).
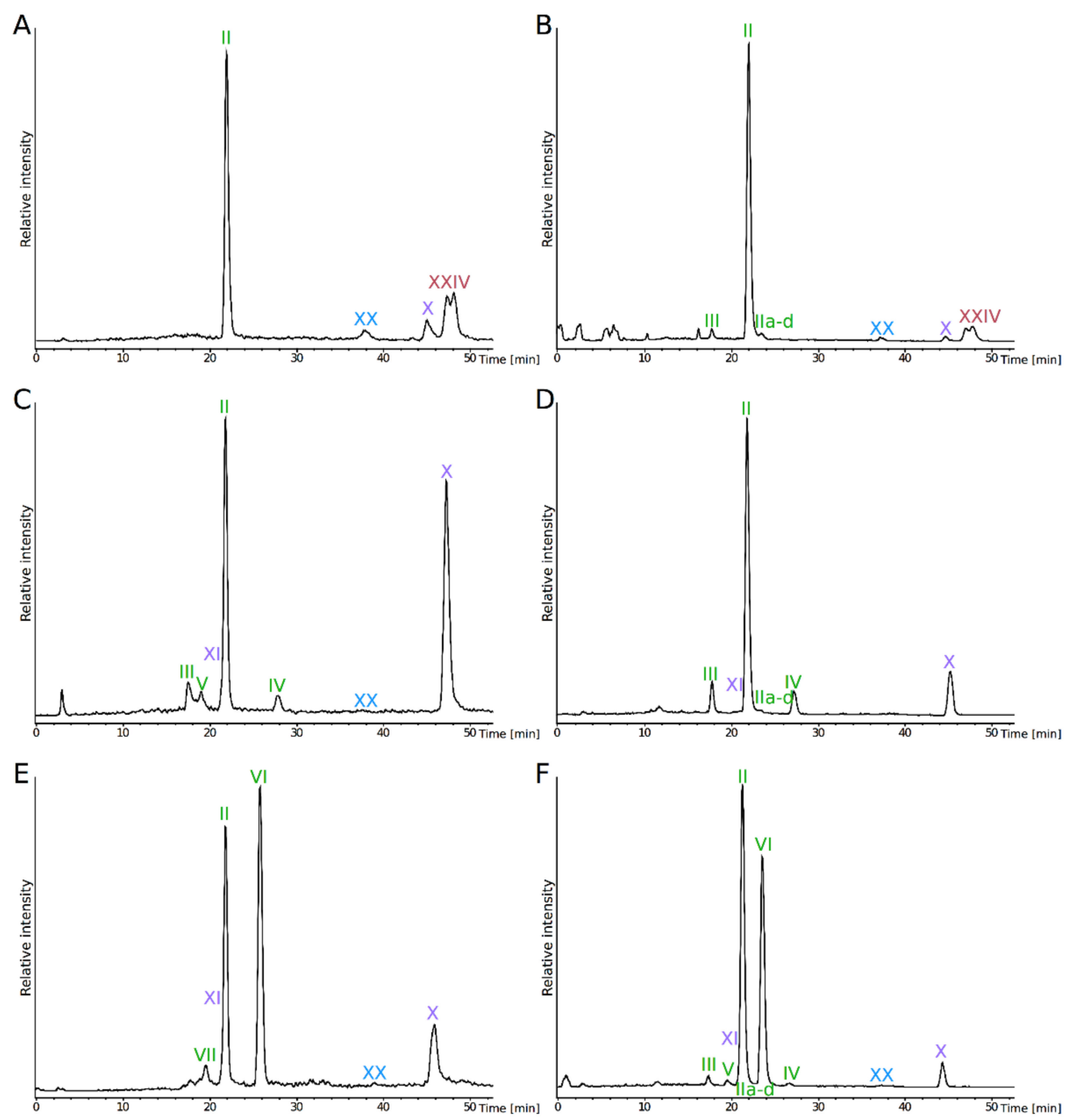

Figure 2. HPLC-ESI-MS chromatograms of intact polar lipids of Pyrococcus furiosus (A, B), P. yayanosii (C, D) and Thermococcus kodakarensis (E, F). Intact polar lipids were detected in positive $(\mathbf{A}, \mathbf{C}$ and $\mathbf{E})$ and negative (B, D and F) ion modes. Chromatograms were drawn by extracting the following protonated and deprotonated ions: positive mode: $893,894,895,936,1056,1057,1124,1125,1544,1784,1786$ and 1788; negative mode: 881, 883, 889, 891, 892, 893, 934, 1054, 1055, 1122, 1123, 1542, 1782, 1784, and 1786. Refer to Figure 1 for lipid structures and their corresponding masses. 

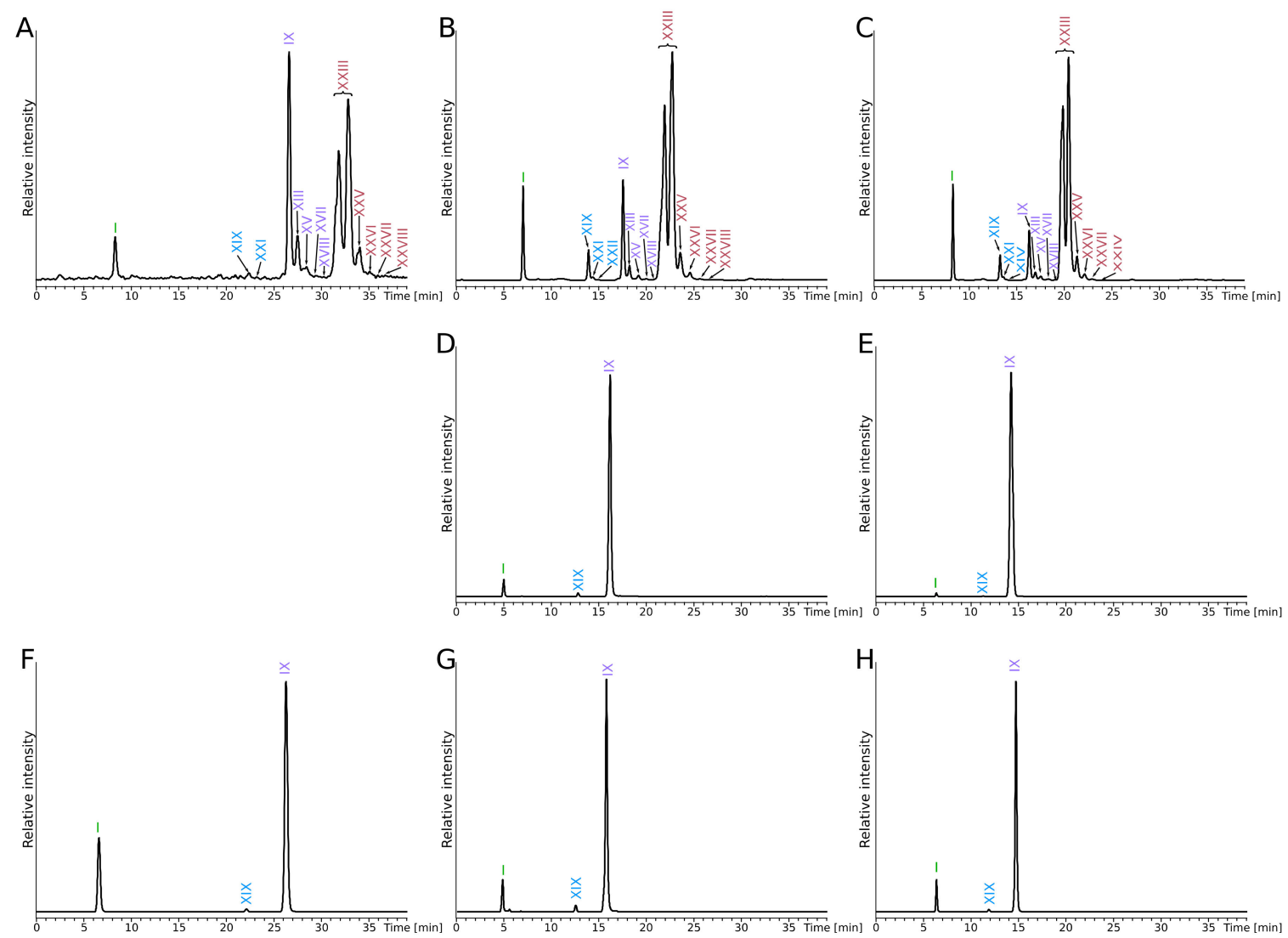

Figure 3. HPLC-APCI-MS chromatograms of core lipids from P. furiosus (A-C), P. yayanosii (D, E) and Thermococcus kodakarensis $(\mathbf{F}-\mathbf{H})$. Total CL (A and $\mathbf{F}$ ) were recovered after direct methanolysis of the cell pellets. Note that the methanolysis of $P$. yayanosii biomass was not performed due to the low amounts of material available for this species. CL from the intact polar lipids (B, D and G) were obtained after methanolysis of the intact polar lipids (IPL) extracts. Residual CL (C, E and H) were recovered after methanolysis of the pre-extracted cells. Chromatograms were drawn by extracting the following protonated ions: 653, 1292, 1294, 1296, 1298, 1300, 1302 and 1304. Refer to Figure 1 for lipid structures and their corresponding masses.

Similarly to P. furiosus, P. yayanosii IPL were dominated by PI-DGD II (ca. 38\% and 61\% in positive and negative ion modes, respectively), together with unsaturated PI-DGD IIa to IId (trace amounts) and PHexNAc-DGD III (ca. 7\% and 10\%) (Figure 2A,C and Table 1). P. yayanosii tetraether lipids were composed of PI-GDGT0-PI X (ca. 44\% and 22\%), with minute amounts of PI-GTGT0-PI XX. A greater diversity of diether-based IPL was observed however in P. yayanosii compared to $P$. furiosus, including DGD with a glycosylated phosphatidyl hexose polar head group (PHexHex-DGD, compound IV, ca. $4 \%$ and $7 \%$ ) and an uncharacterized DGD (compound VI, ca. 5\% in positive ion mode). The latter compound showed a $[\mathrm{M}+\mathrm{H}]^{+}$ion at $m / z$ 1125, which corresponds to that of PHexHex-DGD $\left([\mathrm{M}+\mathrm{H}]^{+}\right.$ 1057) shifted upwards by 68 mass units. Although no fragmentation pattern was available for this compound VI, we suggest that it could be a derivative of PHexHex-DGD bearing an additional isoprene unit attached to the polar head (PHexHex $+\mathrm{C}_{5} \mathrm{H}_{8}$-DGD VI; Figures 1 and $\left.2 \mathrm{C}, \mathrm{D}\right)$. In contrast to P. furiosus, $P$. yayanosii did not exhibit PI-GMGT0-PI but another tetraether-based IPL, which could be identified as PI-GDGT0 XI (ca. 3\% and trace amounts in positive and negative ion modes, respectively).

Thermococcus kodakarensis exhibited the greatest diversity of DGD-based IPL of the three Thermococcales species investigated. It consisted of the same diethers as those from P. yayanosii, with the addition of a derivative of PHexHex-DGD IV $\left([\mathrm{M}+\mathrm{H}]^{+} 1057\right)$, for which one of the hexose group is replaced by a hexosamine moiety (PHexHexNH $\left.\mathrm{PH}_{2} \mathrm{DGD} \mathrm{V},[\mathrm{M}+\mathrm{H}]^{+} 1056\right)$, and an uncharacterized DGD (compound VII; Figure 2E,F). Similarly to PHexHex $+\mathrm{C}_{5} \mathrm{H}_{8}-\mathrm{DGD}$ VI, the protonated molecular 
ion of compound VII $\left([\mathrm{M}+\mathrm{H}]^{+} 1124\right)$ corresponds to that of PHexHexNH${ }_{2}-\mathrm{DGD}\left([\mathrm{M}+\mathrm{H}]^{+} 1056\right)$ shifted upwards by 68 mass units. Compound VII was thus assumed to be a derivative of $\mathrm{PHexHexNH}_{2}-\mathrm{DGD}$ bearing an additional isoprene unit attached to the polar head (PHexHexNH${ }_{2}+\mathrm{C}_{5} \mathrm{H}_{8}$-DGD VII; Figures 1 and $2 \mathrm{E}, \mathrm{F})$. In contrast to both P. furiosus and P. yayanosii, whose IPL were dominated by PI-DGD II, the most abundant IPL of T. kodakarensis was PHexHexNH $\mathrm{N}_{2}$-DGD V (ca. 41\% and 42\%), although high amounts of PI-DGD II (ca. 31\% and 51\%) were also recovered (Figure 2E,F and Table 1). PI-GDGT0-PI $X$ and PI-GDGT0 XI represented respectively ca. 14\% and $9 \%$ of T. kodakarensis IPL in the positive ion mode and ca. $3 \%$ and $2 \%$ in the negative ion mode, respectively (Table 1 ).

In agreement with its IPL composition, the CL recovered from $P$. furiosus showed a large diversity of structures, which included DGD I, GTGT0 XIX, GDGT0 IX and GMGT0 XXIII (Figure 3A-C). However, a variety of cyclopentane ring-containing tetraethers, including GTGT with 1 and 2 rings (GTGT1 and 2, XXI and XXII, respectively), GDGT with 1 to 4 rings (GDGT1 to 4, XIII, XV, XVII and XVIII, respectively) and GMGT with 1 to 4 rings (GMGT1 to 4, XXV to XXIIIV, respectively), was obtained upon acidic methanolysis of $P$. furiosus lipid extract, pre-extracted cells and biomass (Figure $3 \mathrm{~A}-\mathrm{C}$ ). Correcting for the response factor for diethers relative to tetraethers [26], we determined that the most abundant CL in the three different methanolysates of $P$. furiosus were DGD I (ca. 44\%, 45\% and 36\%, respectively) and GMGT0 XXIII (ca. 41\%, 43\% and 33\%, respectively) (Table 2). GDGT0 IX was present in relatively high abundance only in the methanolysate of the intact biomass (ca. 17\%), whereas methanolysis of the lipid extract and of the pre-extracted cells both yielded rather low proportions of it (ca. $6 \%$ and $4 \%$, respectively). Although GTGT and ring-containing tetraethers were detected in the three CL extracts, they were systematically recovered in low abundances, i.e., below 5\% (Table 2). In contrast to P. furiosus, the CL recovered from P. yayanosii and T. kodakarensis exhibited a low diversity of core structures, consisting exclusively of DGD I, GTGT0 XIX and GDGT0 IXV (Figure 3D-H). GDGT0 IX was the most abundant CL in P. yayanosii extracts, reaching ca. $64 \%$ and $92 \%$ in the methanolysates of the IPL and of the pre-extracted cells, respectively (Table 2). Methanolysis of the biomass of T. kodakarensis yielded more diethers than tetraethers, i.e., ca. 56\% and 43\%, respectively, whereas that of its IPL and pre-extracted cells released a slight majority of tetraethers (ca. 61\% and 50\%, respectively; Table 2). 
Table 1. Intact polar lipids (IPL) composition (relative \%) of Pyrococcus furiosus, Pyrococcus yayanosii and Thermococcus kodakarensis.

\begin{tabular}{|c|c|c|c|c|c|c|c|c|c|c|c|}
\hline \multirow{4}{*}{ Species } & \multirow{4}{*}{$\begin{array}{c}\text { MS } \\
\text { Mode }\end{array}$} & \multicolumn{6}{|c|}{ Diethers* } & \multicolumn{4}{|c|}{ Tetraethers * } \\
\hline & & \multirow{2}{*}{$\begin{array}{c}\text { DGDsat+uns } \\
\text { PI }\end{array}$} & \multicolumn{5}{|c|}{ DGD } & \multicolumn{2}{|c|}{ GDGT0 } & \multirow{2}{*}{$\begin{array}{c}\text { GTGT0 } \\
\text { PI+PI } \\
\end{array}$} & \multirow{2}{*}{$\begin{array}{c}\text { GMGT0 } \\
\text { PI+PI }\end{array}$} \\
\hline & & & PHexNAc & PHexHex & $\mathrm{PHexHexNH}_{2}$ & $\mathrm{PHexHex}+\mathrm{C}_{5} \mathrm{H}_{8}$ & $\mathrm{PHexHexNH}_{2}+\mathrm{C}_{5} \mathrm{H}_{8}$ & PI+PI & PI & & \\
\hline & & II+IIa+IIb+IIc+IId & III & IV & V & VI & VII & IX & $x$ & $X X$ & XXIV \\
\hline \multirow{2}{*}{ P. furiosus } & ESI+ & 59 & ND & ND & ND & ND & ND & 8 & ND & 5 & 28 \\
\hline & ESI- & 84 & 2 & ND & ND & ND & ND & 2 & ND & 1 & 11 \\
\hline \multirow{2}{*}{ P. yayanosii } & ESI+ & 38 & 7 & 4 & ND & 5 & ND & 44 & 3 & Traces & ND \\
\hline & ESI- & 61 & 9 & 7 & ND & ND & ND & 22 & Traces & ND & ND \\
\hline \multirow{2}{*}{ T. kodakarensis } & ESI+ & 31 & ND & ND & 41 & ND & 5 & 14 & 9 & Traces & ND \\
\hline & ESI- & 51 & 1 & Traces & 42 & Traces & ND & 3 & 2 & Traces & ND \\
\hline
\end{tabular}

* Relative proportions account for protonated, ammoniated and sodiated adducts in positive mode and for deprotonated adducts in negative mode, and were calculated assuming a response factor of 1 for all IPL (see methods). Traces, <1\%. ND: not detected.

Table 2. Core lipid composition (relative \%) of the total cell pellet (totCL), the total lipid extract (CLfromIPL) and the pre-extracted pellet (resCL) of Pyrococcus furiosus, P. yayanosii and Thermococcus kodakarensis.

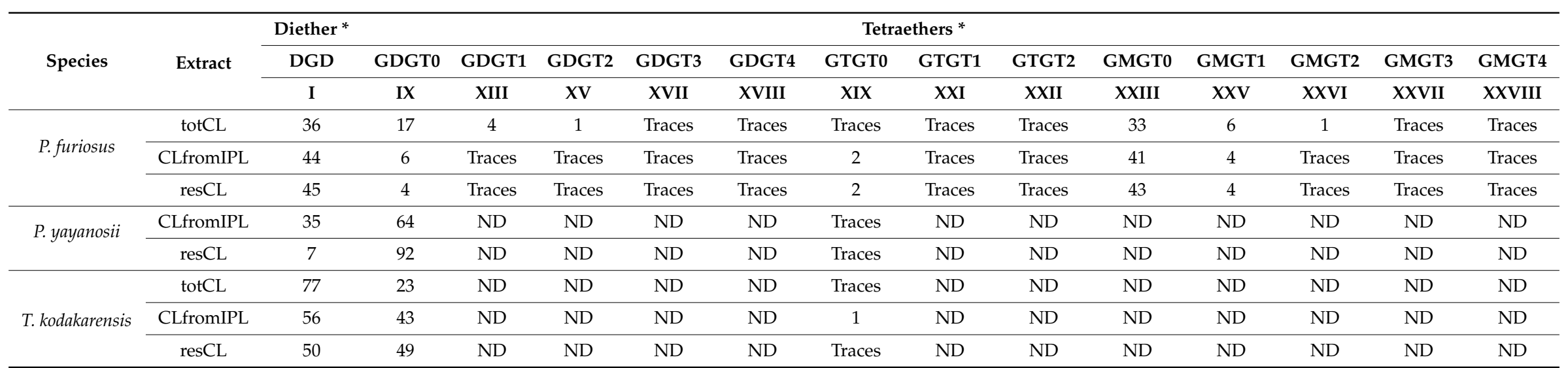

* Relative proportions account for protonated adducts only and were calculated using a response factor of $1 / 10$ for DGD I relative to tetraether lipids (see methods). Traces, $<1 \%$. ND: not detected. 


\section{Discussion}

Our study reports the IPL and CL compositions of Pyrococcus furiosus, Thermococcus kodakarensis and, for the first time, that of the obligate piezophile P. yayanosii. Since the IPL composition of T. kodakarensis was recently reported by Meador et al. [29], who coupled a B\&D with trichloroacetic acid extraction with HPLC-ESI-MS analysis, this species represented a control of our lipid extraction procedure and membrane lipid analysis. Under our analytical conditions, T. kodakarensis exhibited the most diverse IPL composition of the three species analyzed, i.e., PI-DGD II, PHexNAc-DGD III, PHexHex-DGD IV,

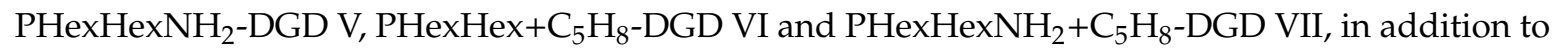
the tetraether-based IPL PI-GTGT0-PI XX, PI-GDGT0-PI X and PI-GDGT0 XI (Figure 2E,F). These IPL represented the major IPL previously recovered by Meador et al. [29] using comparable extraction and analytical methods and under similar growth conditions. Only the minor IPL detected by Meador et al. [29], i.e., glycolipids, aminoacid-based and phosphatidylglycerol(PG)-based lipids, altogether representing less than $10 \%$ of $T$. kodakarensis IPL, were not detected here. Thus, our extraction and analytical procedures prove reliable in describing the dominant IPL of Thermococcales species.

The IPL composition of the obligate piezophilic archaeon P. yayanosii consisted of PI-DGD II, PHexNAc-DGD III, PHexHex-DGD IV, PHexHexPI+C ${ }_{5} \mathrm{H}_{8}$-DGD VI, PI-GTGT0-PI XX, PI-GDGT0-PI X and PI-GDGT0 XI (Figures 1 and 2C,D). In comparison, P. furiosus displayed a lower diversity of polar head groups, i.e., PI and PHexNAc, but attached to a much wider variety of CL, i.e., DGD, GDGT0, GTGT0 and GMGT0 (Figures 1 and 2A,B). Under our analytical conditions, P. yayanosii did not exhibit specific IPL compared to its piezosensitive relatives. Our results thus suggest that the adaptation to high hydrostatic pressures does not involve specific core structure, but might instead be supported by particular polar head groups that are not detected by our procedure, or by other molecules such as apolar polyisoprenoids as proposed by Cario et al. [11].

Only five IPL, i.e., PI-DGD II, P-HexNAc III, PI-GTGT0-PI XX, PI-GDGT0-PI X and PI-GMGT0-PI XXIV (Figures 1 and 2A,B), were unambiguously detected in our P. furiosus lipid extract. This IPL composition contrasts with those previously reported for this species, although PI-DGD II was systematically the most abundant IPL detected [21,22]. GMGT-based CL [4,25,26] and IPL [33] have been identified in numerous archaea, including some Thermococcales but, to the best of our knowledge, our study constitutes the first identification of GMGT-based lipids in P. furiosus and the first report of PI-GMGT0-PI XXIV and PI-GTGT0-PI XX as archaeal IPL. By contrast, Sprott et al. [21] tentatively identified PG-based IPL, such as PG-DGD VIII and dihexose(2Hex)-GDGT0-PG XII (Figure 1), in addition to PI-DGD II and PHexNAc-DGD III. They also reported the presence of PI-GDGT0 XI, a compound that we did identify in P. yayanosii and T. kodakarensis but not in P. furiosus (Figure 2). Such divergence in the detection of PI-GDGT0 XI between studies suggests either that it is a biosynthetic intermediate between core GDGT0 IX and PI-based tetraethers, or that it is a partial hydrolysis product of other IPL, such as PI-GDGT0-PI X. More recently, Lobasso et al. [22] reported saturated and unsaturated PI-DGD II and IId, PHexNAc-DGD III and PI-GDGT0-PI X, and tentatively identified ring-containing GDGT-based IPL, i.e., HexPI-GDGT1 XIV and HexNAcPI-GDGT2 XVI (Figure 1) in P. furiosus. This study also reported for the first time the presence of the diphytanyl glycerol analogue of cardiolipin XXIX in a hyperthermophilic archaeon (Figure 1), whereas this compound is regularly detected in halophilic archaea [34]. The two previous and the present study of P. furiosus membrane lipids thus highlight a large variety of its lipid membrane composition, and place $P$. furiosus as a prime model for future elucidation of archaeal lipid structural and adaptive functions.

The comparison of these three studies also shows that diverging results can be obtained when analyzing the IPL composition of the same species, and several scenarios might be invoked to explain such discrepancies. First, the lipid compositions of closely related strains might greatly diverge from one another. For instance, while the strain used in our study and that of Lobasso et al. [22] was obtained from the DSMZ (though certainly at very different time periods), that of Sprott et al. [21] was provided by one of their colleagues. It is thus likely that the three strains used have diverged, including from the lipid point of view. Second, cultures of 0.10, 0.25 and $300 \mathrm{~L}$ were employed in Sprott 
et al. [21], in the present study and in Lobasso et al. [22], respectively, which would potentially only allow to detect the most abundant IPL in the present study and that of Sprott et al. [21] while the bigger culture volumes in the study of Lobasso [22] allowed to access rarer lipids, such as HexPI-GDGT1 XIV and HexNAcPI-GDGT2 XVI. In our extracts, PHexHex and PHexNAc polar head groups were only observed attached to DGD and not to GDGT core structures (Figure 2A,B), and P. furiosus displayed low proportions of PHexNAc-DGD III only ( $2 \%$ in negative ion mode; Table 1$)$. In parallel, cyclopentane ring-containing GDGT1 XIII and GDGT2 XV were also detected in low proportions among the CL released from intact cells (ca. $4 \%$ and 1\%, respectively; Figure 3A-C and Table 2). Such minute amounts of both polar head groups and core structures probably prevented minor IPL, such as XIV and XVI, from being detected in our study. Third, T. kodakarensis was demonstrated to adapt its membrane lipid distribution to its growth stage and to the medium composition [29]. The growth temperature and medium composition as well as the incubation time employed in our study greatly differed from those used by Sprott et al. [21] and Lobasso et al. [22], and this probably also contributed to the distinct lipid composition unveiled by the different studies. Fourth, it has been shown that even slight changes in the extraction procedure might lead to distinct lipid recovery rates and thus yield IPL fractions with different compositions [10]. Our study (B\&D extraction with a higher proportion of DCM), and those of Sprott et al. [21] (IPL precipitation after classic B\&D extraction) and Lobasso et al. [22] (classic B\&D extraction) employed quite comparable extraction procedures but recovered drastically different IPL compositions from P. furiosus. Fifth, it has been determined that, similarly to extraction procedures, different analytical techniques may induce the preferential detection of some specific classes of lipids over others, e.g., the negative ionization mode enhances the response of PG-based IPL ions compared to the positive mode [35] while matrix-assisted laser desorption/ionization-time of flight (MALDI-TOF) preferentially spots lipids with net charges or high proton affinity [36]. While HPLC-ESI-MS was employed in our study, Lobasso et al. [22] and Sprott et al. [21] investigated total lipid extracts using MALDI-TOF-MS without prior HPLC lipid separation and fast atom bombardment (FAB)-MS, respectively, which potentially also contributed to the lipid composition discrepancies observed between studies. Altogether, these observations illustrate the plethora of parameters that can influence the lipid diversity recovered from pure cultures of a single archaeal species, and argue in favor of extreme cautiousness when dealing and analyzing archaeal lipid data. Similarly to P. furiosus, further reevaluations of the lipid diversity of previously analyzed archaea might help constraining the variability across studies and refine our knowledge of the archaeal lipidome.

To further evaluate our extraction and analytical procedures and determine whether they were able to reveal the majority of IPL from P. furiosus, P. yayanosii and T. kodakarensis, acidic methanolysis was performed on the cell pellets (Figure $3 \mathrm{~A}, \mathrm{~F}$ ), the B\&D extracts (Figure $3 \mathrm{~B}, \mathrm{D}, \mathrm{G}$ ) and the extracted cells (Figure 3C,E,H). First, the methanolysates from residual cells after B\&D extraction revealed the presence of remaining, non-extracted lipids (Figure 3C,E,H and Table 1), showing that the B\&D extraction procedure does not lead to the complete recovery of the lipid content of Thermococcales cells, as already demonstrated in previous studies $[10,11]$. The methanolysates of intact cells of the three species predominantly contained tetraethers, which represented from ca. $50 \%$ up to $93 \%$ of the total CL (Table 1). By contrast, tetraether-based IPL represented from ca. $5 \%$ to $47 \%$ of the lipid extracts of the three strains (Table 2). Such large discrepancies in di- and tetraether distributions between extracted IPL and CL released upon hydrolysis of intact cells may indicate that tetraethers are much more reluctant to extraction by the B\&D procedure than their diether counterparts. The elevated proportions of DGD in the methanolysates of total lipid extracts, ranging from ca. $36 \%$ to $56 \%$ (Table 1), further support this assumption. In addition, the lipid extracts from P. furiosus, P. yayanosii and T. kodakarensis were vastly dominated by PI-based IPL, which represented from ca. $58 \%$ to ca. $98 \%$ of $T$. kodakarensis and P. furiosus lipid extracts in negative ion mode, respectively (Figure 2 and Table 1). It is very unlikely that PI-based phospholipids are the only ones present in these strains, because membranes of divergent lipid compositions are essential in cells to host specific membrane functionalities. Furthermore, we could obtain a P. furiosus mutant in which the genes involved in the specific fixation of the PI polar headgroup 
and derivatives was knocked out, showing that the strain can be viable in the absence of lipids of this class. Thus, in addition to DGD-based IPL, our extraction procedure and LC-MS analysis seem to strongly favor PI-based IPL. We also noted a great inefficiency to fully extract tetraether-based lipids and some diethers, suggesting that the unextracted IPL may harbor polar head groups reluctant to the current extraction methods. Based on the existence of ether-based cardiolipin [22] and covalently bonded lipids to membrane proteins in Archaea [35], such undetected IPL might for instance be involved in intricate lipid-lipid or lipid-protein complexes that are out of our analytical window.

\section{Conclusions}

We reassessed the intact and core lipid compositions of $P$. furiosus and unraveled the presence of 19 membrane lipids in this archaeon, including a variety of core structures, i.e., DGD, GMGT, GDGT, GTGT, and a few polar head groups, i.e., PI and PHexNAc. Combined with the previous studies of Sprott et al. [21] and Lobasso et al. [22], this brings the number of known lipid structures in P. furiosus to at least 25. The CL released upon acid methanolysis of extracted cells suggest that this number might be even greater, and that a vast diversity of $P$. furiosus IPL remains elusive because of difficulties of extraction and/or analysis. Although P. furiosus lipids have been investigated multiple times before, our data further demonstrate that extraction and analytical conditions are of paramount importance and require a great deal of rigor and some more improvements to access and comprehend the complete archaeal lipidome. Altogether, these results illustrate the complexity and uniqueness of the P. furiosus membrane structure and promote this species as a prime model of Thermococcales to elucidate archaeal lipid diversity and functions. The first membrane lipid characterization of P. yayanosii, the most piezophilic Thermococcales member isolated so far, showed a relatively low diversity of CL bearing exclusively phosphosugars and derivatives as polar heads. Our results bring the number of Thermococcales with known IPL diversity to eight, five of which belong to the Pyrococcus genus. The lipid diversity reported here contrasts with the much simpler lipid composition of closely related species, such as P. woesei, which only exhibited PI-DGD [20], and argue in favor of further in-depth (re)investigations of the Thermococcales lipidome to better characterize their lipid diversity and related physiological and adaptive functions.

Author Contributions: Conceptualization, funding acquisition, project administration and supervision, P.M.O., P.S. and V.G.; Formal analysis, M.T. and V.K.; Investigation, A.C., M.T. and V.K.; Methodology, P.S. and V.K.; Visualization and writing - original draft, M.T.; writing-review and editing, M.T., P.M.O., P.S. and V.G. All authors have read and agree to the published version of the manuscript.

Funding: M.T. was supported by a Ph.D. grant from the French Ministry of Research and Technology. This work was funded by a grant from the French National Research Agency (ArchaeoMembranes, ANR-17-CE11-0012-01) and a grand from the CNRS Interdisciplinary program "Origines" (Project ReseArch).

Conflicts of Interest: The authors declare no conflict of interest

\section{Abbreviations}

$\begin{array}{ll}\text { IPL } & \text { Intact polar lipid(s) } \\ \text { CL } & \text { Core lipid(s) } \\ \text { MeOH } & \text { Methanol } \\ \text { DCM } & \text { Dichloromethane } \\ \text { HPLC } & \text { High-performance liquid chromatography } \\ \text { MS } & \text { Mass spectrometry } \\ \text { ESI } & \text { Electrospray ionization } \\ \text { APCI } & \text { Atmospheric-pressure chemical ionization } \\ \text { MGD } & \text { Monoalkyl glycerol diethers } \\ \text { DGD } & \text { Dialkyl glycerol diethers } \\ \text { GDGT } & \text { Glycerol dialkyl glycerol tetraethers } \\ \text { GTGT } & \text { Glycerol trialkyl glycerol tetraethers } \\ \text { GMGT } & \text { Glycerol monoalkyl glycerol tetraethers }\end{array}$


B\&D Bligh and Dyer

PI Phosphatidyl inositol

PHexNAc Phosphatidyl N-acetylhexosamine

PHexHex Glycosylated phosphatidyl hexose

PG Phosphatidyl glycerol

FAB Fast atom bombardment

MALDI-TOF Matrix-assisted laser desorption ionization-time of flight

\section{References}

1. Baba, T.; Toshima, Y.; Minamikawa, H.; Hato, M.; Suzuki, K.; Kamo, N. Formation and characterization of planar lipid bilayer membranes from synthetic phytanyl-chained glycolipids. BBA Biomembr. 1999, 1421, 91-102. [CrossRef]

2. Komatsu, H.; Chong, P.L.-G. Low permeability of liposomal membranes composed of bipolar tetraether lipids from thermoacidophilic archaebacterium Sulfolobus acidocaldarius. Biochemestry 1998, 37, 107-115. [CrossRef] [PubMed]

3. Comita, P.B.; Gagosian, R.B.; Pang, H.; Costello, C.E. Structural elucidation of a unique macrocyclic membrane lipid from a new, extremely thermophilic, deep-sea hydrothermal vent archaebacterium, Methanococcus jannaschii. J. Biol. 1984, 259, 15234-15241.

4. Knappy, C.S.; Nunn, C.E.M.; Morgan, H.W.; Keely, B. The major lipid cores of the archaeon Ignisphaera aggregans: Implications for the phylogeny and biosynthesis of glycerol monoalkyl glycerol tetraether isoprenoid lipids. Extremophiles 2011, 15, 517-528. [CrossRef] [PubMed]

5. Nichols, D.S.; Miller, M.R.; Davies, N.W.; Goodchild, A.; Raftery, M.; Cavicchioli, R. Cold adaptation in the Antarctic archaeon Methanococcoides burtonii involves membrane lipid unsaturation. J. Bacteriol. Res. 2004, 186, 8508-8515. [CrossRef]

6. Gambacorta, A.; Trincone, A.; Nicolaus, B.; Lama, L.; De Rosa, M. Unique features of lipids of Archaea. Syst. Appl. Microbiol. 1993, 16, 518-527. [CrossRef]

7. Becker, K.W.; Elling, F.J.; Yoshinaga, M.Y.; Söllinger, A.; Urich, T.; Hinrichs, K.-U. Unusual butane- and pentanetriol-based tetraether lipids in Methanomassiliicoccus luminyensis, a representative of the seventh order of methanogens. Appl. Environ. Microbiol. 2016, 82, 4505-4516. [CrossRef]

8. Jahn, U.; Summons, R.; Sturt, H.; Grosjean, E.; Huber, H. Composition of the lipids of Nanoarchaeum equitans and their origin from its host Ignicoccus sp. strain KIN4/I. Arch. Microbiol. 2004, 182, 404-413. [CrossRef]

9. Koga, Y.; Akagawa-Matsushita, M.; Ohga, M.; Nishihara, M. Taxonomic significance of the distribution of component parts of polar ether lipids in methanogens. Syst. Appl. Microbiol. 1993, 16, 342-351. [CrossRef]

10. Huguet, C.; Martens-Habbena, W.; Urakawa, H.; Stahl, D.A.; Ingalls, A.E. Comparison of extraction methods for quantitative analysis of core and intact polar glycerol dialkyl glycerol tetraethers (GDGTs) in environmental samples. Limnol. Oceanogr. Meth. 2010, 8, 127-145. [CrossRef]

11. Cario, A.; Grossi, V.; Schaeffer, P.; Oger, P.M. Membrane homeoviscous adaptation in the piezo-hyperthermophilic archaeon Thermococcus barophilus. Front. Microbiol. 2015, 6, 1152-1164. [CrossRef] [PubMed]

12. Nishihara, M.; Koga, Y. Extraction and Composition of polar lipids from the archaebacterium, Methanobacterium thermoautotrophicum: Effective extraction of tetraether lipids by an acidified solvent. J. Biochem. 1987, 101, 997-1005. [CrossRef] [PubMed]

13. Yoshinaga, M.Y.; Gagen, E.J.; Wörmer, L.; Broda, N.K.; Meador, T.B.; Wendt, J.; Thomm, M.; Hinrichs, K.U. Methanothermobacter thermautotrophicus modulates its membrane lipids in response to hydrogen and nutrient availability. Front. Microbiol. 2015, 6, 5-14. [PubMed]

14. Marteinsson, V.T.; Birrien, J.L.; Reysenbach, A.L.; Vernet, M.; Marie, D.; Gambacorta, A.; Messner, P.; Sleytr, U.B.; Prieur, D. Thermococcus barophilus sp. nov., a new barophilic and hyperthermophilic archaeon isolated under high hydrostatic pressure from a deep-sea hydrothermal vent. Int. J. Syst. Evol. Micr. 1999, 49, 351-359.

15. Fiala, G.; Stetter, K.O. Pyrococcus furiosus sp. nov. represents a novel genus of marine heterotrophic archaebacteria growing optimally at 100 C. Arch. Microbiol. 1986, 145, 56-61. 
16. Farkas, J.; Stirrett, K.; Lipscomb, G.L.; Nixon, W.; Scott, R.; Adams, M.W.W.; Westpheling, J. Recombinogenic properties of Pyrococcus furiosus strain COM1 enable rapid selection of targeted mutants. Appl. Environ. Microbiol. 2012, 78, 4669-4676. [CrossRef]

17. Lipscomb, G.L.; Stirrett, K.; Schut, G.J.; Yang, F.; Jenney, F.E.; Scott, R.A.; Adams, M.W.; Westpheling, J. Natural competence in the hyperthermophilic archaeon Pyrococcus furiosus facilitates genetic manipulation: Construction of markerless deletions of genes encoding the two cytoplasmic hydrogenases. Appl. Environ. Microbiol. 2011, 77, 2232-2238. [CrossRef]

18. Zillig, W.; Holz, I.; Klenk, H.-P.; Trent, J.; Wunderl, S.; Janekovic, D.; Imsel, E.; Haas, B. Pyrococcus woesei, sp. nov., an ultra-thermophilic marine archaebacterium, representing a novel order, Thermococcales. Syst. Appl. Microbiol. 1987, 9, 62-70. [CrossRef]

19. Kanoksilapatham, W.; Gonzalez, J.M.; Maeder, D.L.; DiRuggiero, J.; Robb, F. A proposal to rename the hyperthermophile Pyrococcus woesei as Pyrococcus furiosus subsp. woesei. Archaea 2004, 1, 277-283. [CrossRef]

20. Lanzotti, V.; Trincone, A.; Nicolaus, B.; Zillig, W.; De Rosa, M.; Gambacorta, A. Complex lipids of Pyrococcus and AN1, thermophilic members of archaebcteria belonging to Thermococcales. Biochim. Biophys. Acta Lipids Lipid Metab. 1989, 1004, 44-48. [CrossRef]

21. Sprott, G.D.; Agnew, B.J.; Patel, G.B. Structural features of ether lipids in the archaeobacterial thermophiles Pyrococcus furiosus, Methanopyrus kandleri, Methanothermus fervidus, and Sulfolobus acidocaldarius. Can. J. Microbiol. 1997, 43, 467-476. [CrossRef]

22. Lobasso, S.; Lopalco, P.; Angelini, R.; Vitale, R.; Huber, H.; Müller, V.; Corcelli, A. Coupled TLC and MALDI-TOF/MS analyses of the lipid extract of the hyperthermophilic archaeon Pyrococcus furiosus. Archaea 2012, 2012, 1-10. [CrossRef] [PubMed]

23. Reysenbach, A.-L.; Godfroy, A.; Meunier, J.-R.; Crump, B.C.; Baross, J.; Marteinsson, V.; Barbier, G.; Pace, N.; Prieur, D. Pyrococcus abyssi sp. nov., a new hyperthermophilic archaeon isolated from a deep-sea hydrothermal vent. Arch. Microbiol. 1993, 160, 338-349. [CrossRef]

24. Sugai, A.; Masuchi, Y.; Uda, I.; Itoh, T.; Itoh, Y.H. Core lipids of hyperthermophilic archaeon, Pyrococcus horikoshii OT3. JOSAP 2000, 49, 695-700. [CrossRef]

25. Sugai, A.; Uda, I.; Itoh, Y.H.; Itoh, T. The core lipid composition of the 17 strains of hyperthermophilic archaea. J. Oleo Sci. 2004, 53, 41-44. [CrossRef]

26. Tourte, M.; Schaeffer, P.; Grossi, V.; Oger, P.M. Functionalized membrane domains: An ancestral feature of Archaea? Front. Microbiol. 2020, 11, 526-540. [CrossRef]

27. Callac, N.; Schaeffer, P.; Lesongeur, F.; Rattray, J.E.; Vannier, P.; Michoud, G.; Beauverger, M.; Gayet, N.; Rouxel, O.; Jebbar, M.; et al. Pyrococcus kukulkanii sp. nov., a hyperthermophilic, piezophilic archaeon isolated from a deep-sea hydrothermal vent. Int. J. Syst. Evol. Microbiol. 2016, 66, 3142-3149. [CrossRef]

28. Zeng, X.; Birrien, J.-L.; Fouquet, Y.; Cherkashov, G.; Jebbar, M.; Querellou, J.; Oger, P.; Cambon-Bonavita, M.-A.; Xiao, X.; Prieur, D. Pyrococcus CH1, an obligate piezophilic hyperthermophile: Extending the upper pressure-temperature limits for life. ISME J. 2009, 3, 873-876. [CrossRef]

29. Meador, T.B.; Gagen, E.J.; Loscar, M.E.; Goldhammer, T.; Yoshinaga, M.Y.; Wendt, J.; Thomm, M.; Hinrichs, K.U. Thermococcus kodakarensis modulates its polar membrane lipids and elemental composition according to growth stage and phosphate availability. Front. Microbiol. 2014, 5, 10-23. [CrossRef]

30. Morikawa, M.; Izawa, Y.; Rashid, N.; Hoaki, T.; Imanaka, T. Purification and characterization of a thermostable thiol protease from a newly isolated hyperthermophilic Pyrococcus sp. Appl. Environ. Microbiol. 1994, 60, 4559-4566. [CrossRef]

31. Bligh, E.G.; Dyer, W.J. A rapid method of total lipid extraction and purification. Can. J. Biochem. Physiol. 1959, 37, 911-917. [CrossRef] [PubMed]

32. Damsté, J.S.S.; Rijpstra, W.I.C.; Hopmans, E.C.; Jung, M.-Y.; Kim, J.-G.; Rhee, S.-K.; Stieglmeier, M.; Schleper, C. Intact polar and core glycerol dibiphytanyl glycerol tetraether lipids of group, I.1a and I.1b Thaumarchaeota in soil. Appl. Environ. Microbiol. 2012, 78, 6866-6874. [CrossRef] [PubMed]

33. Baumann, L.M.; Taubner, R.S.; Bauersachs, T.; Steiner, M.; Schleper, C.; Peckmann, J.; Simon, K.M.; Birgel, D. Intact polar lipid and core lipid inventory of the hydrothermal vent methanogens Methanocaldococcus villosus and Methanothermococcus okinawensis. Org. Geochem. 2018, 126, 33-42. [CrossRef]

34. Corcelli, A. The cardiolipin analogues of Archaea. Biochim. Biophys. Acta Biomembr. 2009, 1788, 2101-2106. [CrossRef] 
35. Hankin, J.A.; Murphy, R.C.; Barkley, R.M.; Gijón, M.A. Ion mobility and tandem mass spectrometry of phosphatidylglycerol and bis (monoacylglycerol) phosphate (BMP). Int. J. Mass Spectrom. 2015, 378, 255-263. [CrossRef]

36. Law, K.P.; Zhang, C.L. Current progress and future trends of mass spectrometry-based ar-chaeal lipidomics. Org. Geochem. 2019, 134, 45-61. [CrossRef]

(C) 2020 by the authors. Licensee MDPI, Basel, Switzerland. This article is an open access article distributed under the terms and conditions of the Creative Commons Attribution (CC BY) license (http://creativecommons.org/licenses/by/4.0/). 